\title{
REFERENCES
}

1. E. Bishop, A generalization of the Stone-Weierstrass theorem, Pacific J. Math. 11 (1961), 777-783.

2. R. C. Buck, Bounded continuous functions on a locally compact space, Michigan Math. J. 5 (1958), 95-104.

3. I. Glicksberg, Bishop's generalized Stone-Weierstrass theorem for the strict topology, Proc. Amer. Math. Soc. 14 (1963), 329-333.

UNIVERSITY OF WISCONSIN AND

UNIVERSITY OF CONNECTICUT

\section{WEAK LIMITS OF POWERS OF A CONTRACTION IN HILBERT SPACE ${ }^{1}$}

S. R. FOGUEL

Let $T$ be an operator, on the Hilbert space $H$, with $\|T\| \leqq 1$. Let

$$
H_{0}=\left\{x \mid \text { weak } \lim T^{n} x=0\right\}, \quad H_{1}=H_{0}^{\perp} .
$$

We shall use the facts, proved in [1], that

1. $x \in H_{0}$ if and only if $\lim \left(T^{n} x, x\right)=0$ (Theorem 3.1).

2. On $H_{1}$ the operator $T$ is unitary (Theorem 1.1).

Given $x \in H$ let $x=x_{0}+x_{1}$, where $x_{0} \in H_{0}$ and $x_{1} \in H_{1}$. The purpose of this note is to find conditions, on the sequence $\left(T^{n} x, x\right)$, that will imply that $x_{1}$ is generated by eigenvectors of $T$. This is related to the notion of mixing for ergodic transformations.

THEOREM 1. Let $y$ be in the subspace generated by $T^{n} x, T^{*_{n}} x$, $n=1,2, \cdots$. If $\lim \left(T^{n} y, x\right)=0$, then weak $\lim T^{n} y=0$.

Proof. Since $H_{0}$ and $H_{1}$ are invariant under $T$, it is enough to prove the theorem for the case when $x \in H_{1}$ (and thus also $y \in H_{1}$ ). If $n_{i}$ is any subsequence of the integers and $z=$ weak $\lim T^{n i y}$, then $z$ is orthogonal to $T^{k} x, k=0, \pm 1, \pm 2, \cdots$ (since $T$ is unitary on $H_{1}$ ). But $z$ belongs to the subspace generated by $T^{ \pm n} x$. Hence, $z=0$ and, thus, weak $\lim T^{n} y=0$.

Corollary. Let $P(\lambda)$ be a polynomial whose roots of modulus one are $\lambda_{1}, \cdots, \lambda_{k}$. If $\lim \left(T^{n} P(T) x, x\right)=0$, then $x=x_{0}+x_{1}$ where: $x_{0} \in H_{0}$, $x_{1}=\sum_{i=1}^{k} z_{i}, T z_{i}=\lambda_{i} z_{i}$.

Proof. Let $x=x_{0}+x_{1}$, where $x_{0} \in H_{0}$ and $x_{1} \in H_{1}$. By Theorem 1,

Received by the editors March 25, 1964.

1 This work was partially supported by NSF Grants GP87 and G14736 at Northwestern University. 
$P(T) x \in H_{0}$ and, thus, $P(T) x_{1} \in H_{0}$ or $P(T) x_{1}=0$. The theorem now follows from the spectral representation of $T$ on $H_{1}$.

Consider the example: $H=L_{2}([0,2 \pi), \mu)$ and $(T f)(\theta)=e^{i \theta} f(\theta)$. Then

$$
\left(T^{n} x, x\right)=\int_{0}^{2 \pi} e^{i n \theta}|f(\theta)|^{2} \mu(d \theta)
$$

are the Fourier coefficients of $|f|^{2} \mu$. Thus if

$$
a_{0}\left(T^{n} x, x\right)+a_{1}\left(T^{n+1} x, x\right)+\cdots+a_{p}\left(T^{n+p} x, x\right) \underset{n \rightarrow \infty}{\longrightarrow} 0
$$

then

$$
f=f_{0}+f_{1}, \quad \int e^{i n \theta}\left|f_{0}(\theta)\right|^{2} \mu(d \theta) \rightarrow 0
$$

and

$$
f_{1}=0 \text { except for the points } \theta_{1}, \cdots, \theta_{k} \text {, which }
$$$$
\text { are the roots of } a_{0}+a_{1} e^{i \theta}+\cdots+a_{p} e^{i p \theta}=0 \text {. }
$$

Consider, for any contraction $T$, the polynomial $\lambda-1$ :

If $\lim \left(\left(T^{n} x, x\right)-\left(T^{n-1} x, x\right)\right)=0$, then $\lim \left(T^{n} x, x\right)$ exists. In fact, weak $\lim T^{n} x$ exists since $x=x_{0}+x_{1}$, where $T x_{1}=x_{1}$.

In [2] Sucheston proved that if $T$ is given by an ergodic transformation on a measure space and $\lim \left(\left(T^{n} x, x\right)-\left(T^{n-1} x, x\right)\right)=0$ for $n$ outside of a lacunary sequence and for every $x \in H$, then $T$ is strongly mixing. In our treatment we cannot exclude a lacunary sequence but $T$ can be any contraction, and the condition is assumed for one $x$ only.

Let $g(\theta)=f\left(e^{i \theta}\right)$ be a continuous function of bounded variation. Let $\alpha_{k}$ be the Fourier coefficients of $g$. It is known that the Fourier sums converge uniformly to $g$.

Lemma A. Let $U$ be a unitary operator; then $\left(U^{n} f(U) x, x\right)$ $=\sum_{k=\infty}^{\infty}\left(U^{n+k} x, x\right) \alpha_{k}$.

Proof. Let $E(\theta)$ be the spectral measure of $U, 0 \leqq \theta<2 \pi$. Then

$$
\left(U^{n} f(U) x, x\right)=\int_{0}^{2 \pi} g(\theta) e^{i n \theta}(E(d \theta) x, x)=\sum_{k=-\infty}^{\infty} \alpha_{k} \int_{0}^{2 \pi} e^{i(n+k) \theta}(E(d \theta) x, x) .
$$

Lemma B. The sum $\sum_{k=-\infty}^{\infty}\left(T^{n+k} x, x\right) \alpha_{k}$ is convergent and if $x_{0} \in H_{0}$ then

$$
\sum_{k=-\infty}^{\infty}\left(T^{n+k} x_{0}, x_{0}\right) \alpha_{k} \underset{n \rightarrow \infty}{\longrightarrow} 0
$$


Proof. Let $U$ be the unitary dilation of $T$, in the sense of [3]. Then

$$
\sum_{k=-\infty}^{\infty}\left(T^{n+k} x, x\right) \alpha_{k}=\sum_{k=-\infty}^{\infty}\left(U^{n+k} x, x\right) \alpha_{k}=\left(U^{n} f(U) x, x\right) .
$$

Now if weak $\lim T^{n} x_{0}=0$, then $\left(U^{n} x_{0}, x_{0}\right)=\left(T^{n} x_{0}, x_{0}\right) \rightarrow 0$, hence, weak $\lim U^{n} x=$ weak $\lim U^{*_{n}} x=0$. Thus

$$
\left(U^{n} f(U) x, x\right)=\left(f(U) x, U^{* n} x\right) \rightarrow 0 .
$$

ThEOREM 2. Let $g$ vanish on at most a countable set. If

$$
\lim _{n \rightarrow \infty} \sum_{k=-\infty}^{\infty}\left(T^{n+k} x, x\right) \alpha_{k}=0
$$

then $x=x_{0}+x_{1}$, where $x_{0} \in H_{0}$ and $x_{1}$ is generated by eigenvectors of $T$.

Proof. Let $x=x_{0}+x_{1}$, where $x_{0} \in H_{0}$ and $x_{1} \in H_{1}$. Now

$$
\sum_{k=-\infty}^{\infty}\left(T^{n+k} x, x\right) \alpha_{k}=\sum_{k=-\infty}^{\infty}\left(T^{n+k} x_{0}, x_{0}\right) \alpha_{k}+\sum_{k=-\infty}^{\infty}\left(T^{n+k} x_{1}, x_{1}\right) \alpha_{k} .
$$

By Lemma B the first sum tends to zero; thus $\sum_{k=-\infty}^{\infty}\left(T^{n+k} x_{1}, x_{1}\right) \alpha_{k}$ $\rightarrow 0$. Hence, by Lemma A and Theorem $1, f(T) x_{1}=0$. The theorem now follows from the spectral representation of $T$ on $H_{1}$.

\section{BIBLIOGRAPHY}

1. S. R. Foguel, Powers of a contraction in Hilbert space, Pacific J. Math. 13 (1963), 551-562.

2. L. Sucheston, An ergodic application of almost convergence sequences, Duke Math. J. 30 (1963), 417-422.

3. B. Sz-Nagy, Sur les contractions de l'espace de Hilbert, Acta Sci. Math. 15 (1953), 87-92.

\section{NORTHWESTERN UNIVERSITY AND}

Hebrew UNIVERSity, JERUSALEM, ISRAEL 